\title{
Le rôle des comités d'hygiène, de sécurité et des conditions de travail en France
}

\author{
Une analyse empirique
}

Can workers representatives protect occupational health? An empirical analysis

of the role of health and safety committees (HSWCCS) in France

\section{Thomas Coutrot}

\section{CpenEdition}

Journals

\section{Édition électronique}

URL : http://journals.openedition.org/travailemploi/4108

DOI : 10.4000/travailemploi.4108

ISSN : 1775-416X

\section{Éditeur}

DARES - Ministère du Travail

\section{Édition imprimée}

Date de publication : 30 mars 2009

Pagination : 25-38

ISSN : 0224-4365

\section{Référence électronique}

Thomas Coutrot, "Le rôle des comités d'hygiène, de sécurité et des conditions de travail en France », Travail et Emploi [En ligne], 117 | janvier-mars 2009, mis en ligne le 30 mars 2011, consulté le 10 décembre 2020. URL : http://journals.openedition.org/travailemploi/4108 ; DOI : https://doi.org/ 10.4000/travailemploi.4108 


\title{
Le rôle des comités d'hygiène, de sécurité et des conditions de travail en France: une analyse empirique
}

\author{
Thomas Coutrot $\left({ }^{*}\right)$
}

L'article examine empiriquement, sur données françaises, deux questions : quels sont les déterminants de la présence de représentants des salariés en matière de santé-sécurité (comité d'hygiène, de sécurité et des conditions de travail - CHSCT), et quel est l'impact de cette présence sur la santé des salariés. La présence d'un CHSCT dépend notamment de la présence syndicale et du rapport de forces obtenu par les salariés dans l'établissement, ainsi que du niveau des risques liés au travail. La présence d'un CHSCT apparaît associée à une meilleure qualité des politiques de prévention de l'établissement, toutes choses égales par ailleurs, pour les risques chimiques et biologiques mais pas les risques physiques et organisationnels. On n'observe pas de lien entre la présence de CHSCT et la santé des salariés.

Depuis une vingtaine d'années, le nombre de maladies professionnelles reconnues chaque année a connu en France une croissance exponentielle, passant de moins de 5000 à la fin des années 1980 à plus de 40000 en 2006, dont 2000 nouveaux cas de cancers professionnels et près de 30000 cas de troubles musculo-squelettiques. L'ampleur du problème de santé publique dépasse d'ailleurs largement ces chiffres, puisque les maladies professionnelles sont très largement sous-déclarées, comme le reconnaissent tous les acteurs de la santé au travail (ThÉBAUD-MoNY, 1991). La santé au travail constitue désormais un enjeu majeur des politiques sanitaires.

Le renforcement de l'action des représentants du personnel en matière de santé-sécurité (RP-SS) pourrait-il contribuer efficacement à la préservation de la santé des travailleurs? Il existe sur ce thème une abondante littérature scientifique internationale. La majorité des travaux - mais pas tous - concluent à l'existence d'une certaine influence positive de la représentation du personnel (WALTERS, FrICK, 2000).

On peut distinguer deux types d'études présentant des résultats favorables: les premières «montrent que les indicateurs objectifs de performance en matière de santé-sécurité, comme par exemple les taux d'accidents du travail, sont améliorés dans les situations où existent des dispositifs de concertation et/ou les syndicats assurent la représentation

Dares, ministère du Travail : thomas.coutrot@travail.gouv.fr des salariés dans l'établissement» (WALTERs, 2003, p. 9). Dans ces études, on peut donc observer un lien direct et positif entre la présence d'une représentation du personnel en matière de santé-sécurité et la santé des salariés. La deuxième catégorie de travaux montre «un lien entre la présence de structures représentatives et les indicateurs d'un engagement plus systématique du management dans des politiques de prévention» (WaLters, ibidem). Des liens empiriques sont alors établis non pas directement entre la représentation du personnel et une meilleure santé des travailleurs, mais entre la représentation et des politiques managériales de prévention plus actives, repérées par des indicateurs comme l'existence d'une évaluation des risques, une préoccupation plus forte de la part des managers et/ou des salariés pour la prévention des risques, une activité plus importante des services de prévention ..., indicateurs dont on peut raisonnablement espérer qu'ils induisent une meilleure protection de la santé des salariés.

La littérature comprend des études sur le Royaume-Uni, les USA, l'Australie, le Canada, la Suède, mais pas la France. Dans ce pays, le droit du travail donne une assise solide à la représentation des salariés en matière d'hygiène et de sécurité, puisqu'il prévoit la création dans toutes les entreprises de cinquante salariés ou plus d'un CHSCT doté d'attributions importantes. Mais il n'existe à ce jour aucune étude empirique concernant l'impact des CHSCT sur les politiques de prévention des entreprises françaises et sur la santé-sécurité de leurs salariés. C'est cette lacune que ce papier vise à combler. 


\section{Questions de recherche et données empiriques mobilisées}

On s'appuie ici sur une approche théorique néogramscienne en termes de "politique de production» (Burawoy, 1999), qui décrit le rapport salarial comme le lieu de déploiement de stratégies politiques de domination par lesquelles les directions d'entreprise obtiennent le consentement des travailleurs à leur propre exploitation. Dans ce cadre théorique les mécanismes sous-jacents à l'éventuelle efficacité des RP-SS sont de deux types: des mécanismes d'ordre cognitif (Worker Knowledge) et d'ordre politique (Worker Power) (Hall, Forrest, Sears, CARLAN, 2006).

Les mécanismes d'ordre cognitif renvoient aux enjeux de (mé)connaissance du «travail réel» des salariés, par contraste avec le travail prescrit (GUÉRIN et al., 2001). On sait que le management méconnaît souvent les processus de travail réels mis en œuvre par les salariés pour gagner le «jeu de la production» (BuRAWOY, 1979) et atteindre les objectifs fixés par la direction. Les RP-SS peuvent favoriser l'émergence de la connaissance $\mathrm{du}$ «travail réel» détenue par les salariés, et contribuer ainsi à la conception des politiques de prévention. D'autre part les débats et controverses dans le CHSCT favorisent, à des degrés divers sans doute, l'identification des risques et la prise de conscience par les acteurs (management, préventeurs, représentants du personnel, salariés). Enfin la formation que reçoivent les RP-SS, les expériences accumulées au sein de réseaux syndicaux extérieurs à l'entreprise, peuvent améliorer les capacités collectives d'identification des dangers et de formulation de solutions.

Les mécanismes cognitifs sont essentiels car il ne suffit pas que des risques existent objectivement pour qu'ils soient perçus comme tels, ni pour que des actions de prévention s'ensuivent. Même les salariés exposés à des risques évidents pour l'observateur extérieur, peuvent les dénier pour se protéger de la peur. Dans l'enquête SUMER 2003 ( $c f$. infra), $8 \%$ des salariés ont été exposés à un agent cancérogène au cours de la semaine précédant l'enquête: seulement $38 \%$ d'entre eux disent que leur travail est «plutôt mauvais pour leur santé» et $5 \%$ qu'ils souhaitent «changer de poste ou de travail à cause des risques du travail ». C'est certes plus que pour la moyenne de la population enquêté (respectivement $28 \%$ et $2 \%$ ), mais cela pose néanmoins clairement la question de la prise de conscience ou de «l'objectivation» (GollaC, 1998) des risques.

Les mécanismes d'ordre politique, quant à eux, renvoient plus classiquement à la question du rapport des forces: l'implication des représentants du personnel dans le domaine «santé-sécurité» peut contribuer à focaliser l'action revendicative sur les questions de santé-sécurité, renforçant la pression sur le management pour l'adoption de politiques de prévention efficaces.

Ce rôle du politique exprime le caractère conflictuel du rapport salarial, où les acteurs sont animés de logiques hétérogènes et souvent contradictoires (Coutrot, 2002). Certes il existe souvent une complémentarité entre des politiques managériales actives (investissement dans des actions ergonomiques, recours à des experts internes ou externes pour identifier les risques et développer des politiques préventives) et l'implication active des salariés et de leurs représentants (consultation et mobilisation des salariés, participation compétente aux instances de prévention...) (WaLTERS, FricK, 2000; GARCIA et al., 2007). C'est pourquoi en matière de prévention, activisme managérial et activisme syndical tendent souvent à se renforcer mutuellement plutôt qu'à se neutraliser. Néanmoins, leur perception de leur intérêt économique amène souvent les entreprises à externaliser les coûts des atteintes à la santé au travail, sur le système de protection sociale générale et sur les salariés eux-mêmes, plutôt que de financer des programmes de prévention parfois coûteux. L'intervention des salariés et de leurs représentants peut donc largement influer sur les politiques de prévention mises en œuvre, que ce soit via le conflit, la coopération, ou plus probablement les deux à la fois.

Encore faut-il qu'il existe une représentation du personnel, qu'elle s'occupe de santé-sécurité, et qu'elle le fasse de façon suffisamment autonome par rapport au management pour apporter une contribution spécifique. La plupart des travaux empiriques sur la question indiquent qu'on trouve plus souvent des RP-SS dans les grands établissements que dans les petits, dans l'industrie manufacturière que dans les services. On peut néanmoins s'interroger sur les explications de ces constats, et chercher si ces "effets» de taille ou de secteur ne reflètent pas surtout des impacts de la réglementation, des stratégies managériales ou syndicales ou la présence de risques spécifiques.

Trois questions de recherche orientent l'étude ici présentée. La première concerne les conditions d'émergence d'une représentation des salariés en matière de santé-sécurité: quelles sont les caractéristiques des relations professionnelles sur le lieu de travail qui favorisent l'existence et l'activité d'une telle représentation? La seconde question concerne elle aussi l'émergence d'une représentation mais sous un autre angle: quelle relation y a t-il entre la présence de risques professionnels (et de divers types de risque) et la présence d'une représentation? La dernière question, la plus classiquement traitée dans la littérature évoquée ci-dessus, concerne la relation entre l'existence d'une représentation et la santé au travail: en présence de RP-SS, la prévention des risques professionnels est-elle mieux orga- 
nisée et plus efficace, les salariés sont-ils mieux informés, en meilleure santé?

À propos de cette dernière question, certains observateurs estiment qu'il n'est pas possible «d'isoler un effet du CHSCT de l'ensemble de la politique de prévention conduite par tous les acteurs spécialisés, encore moins d'isoler l'action des CHSCT des développements technologiques, de l'intensification du travail, de l'évolution de la réglementation, des rapports sociaux, du climat politico-social et surtout de l'action des syndicats » (FILOche, 2001, p. 71). Tout en ayant conscience de cette difficulté, il apparaît néanmoins possible de tenter l'exercice grâce au matériau statistique disponible, qui permet de prendre en compte l'influence de nombre des facteurs évoqués par G. Filoche. Pour ce faire on mobilise ici trois grandes enquêtes nationalement représentatives portant sur les conditions de travail et les relations de travail: SUMER 2003, REPONSE 2004 et Conditions de travail 2005. Ces enquêtes permettent de construire des indicateurs originaux concernant aussi bien les conditions d'émergence de la représentation des salariés en matière de santé-sécurité, que la qualité de la prévention des risques et la santé des salariés eux-mêmes.

L'enquête SUMER 2003, réalisée par 1800 médecins du travail auprès de 50000 salariés de tous les secteurs d'activité sauf l'administration publique, identifie pour chaque poste de travail l'ensemble des expositions à des risques professionnels subies par le salarié. Elle fournit également quelques informations sur l'établissement employeur, notamment sur la présence ou non d'un CHSCT; ainsi que sur les dispositifs de prévention mis à la disposition du salarié s'il est exposé à certains risques (bruit, rayonnement, agents chimiques ou biologiques). On demande également au médecin enquêteur son jugement sur la qualité de la prévention sur le poste de travail étudié.

L'enquête REPONSE est la filleule française de l'enquête britannique «WIRS» (Workplace Industrial Relations Survey), et vise, comme sa marraine, à décrire les relations professionnelles en entreprise grâce à des entretiens passés dans un échantillon représentatif d'établissements autour d'un questionnaire fermé, avec les managers et les représentants du personnel. Elle a été menée pour les années 1992, 1998 et 2004, ce qui a permis de mesurer le niveau et l'évolution de l'implantation des institutions représentatives du personnel (et en particulier des CHSCT) en fonction des caractéristiques des établissements. L'enquête fournit de très nombreuses informations sur les politiques de gestion de la main-d'œuvre, l'organisation du travail, les négociations, les conflits. En outre, l'édition de 2004 comporte un questionnaire postal rempli par 8000 salariés, interrogés sur leur perception de leur travail et des relations sociales dans leur établissement. Nous utiliserons ici le volet «manager» et le questionnaire «salarié».

L'enquête Conditions de travail est elle aussi une enquête périodique, menée celle-ci depuis 1978 (avec des éditions intermédiaires en 1984, 1991 et 1998); elle a largement inspiré la première édition de l'enquête européenne sur les conditions de travail, menée par la Fondation européenne pour l'amélioration des conditions de travail et de vie («Fondation de Dublin») tous les cinq ans depuis 1990. L'enquête française de 2005, la plus récente, a été menée auprès de 18000 salariés, et décrit les conditions de travail telles qu'elle sont vécues par les salariés eux-mêmes; elle fournit pour la première fois des informations sur les politiques de préventions des entreprises, en posant aux salariés quelques questions sur les ressources (consignes de sécurité, formation, médecine du travail...) dont ils disposent pour faire face aux risques professionnels qu'ils rencontrent dans leur travail (COUTROT, 2008).

\section{Relations professionnelles et mise en place du CSHCT}

En France la création d'un comité d'hygiène, de sécurité et des conditions de travail (CHSCT) est théoriquement obligatoire dans les établissements employant au moins cinquante salariés. Ce comité est appelé à jouer un rôle important dans la prévention des risques professionnels, puisqu'il doit être associé à l'élaboration du plan de prévention (obligatoire dans toutes les entreprises); en outre il doit en principe être consulté par l'employeur avant tout changement technique ou organisationnel ayant des conséquences sur les conditions de travail. En pratique, les CHSCT ne sont pas présents dans tous les établissements assujettis, puisque selon l'enquête REPONSE 2004, seuls $77 \%$ des établissements de plus de 50 salariés ont effectivement un CHSCT $(95 \%$ pour les établissements de plus de 500 salariés, mais $57 \%$ seulement de ceux qui emploient entre 50 et 100 salariés).

Le CHSCT est composé de membres du comité d'entreprise (CE) spécialement désignés pour cette tâche. Le $\mathrm{CE}$ est une instance représentative du personnel, élue tous les quatre ans dans les établissements de 50 salariés et plus. Dans nombre d'établissements de taille moyenne (entre 50 et 500 salariés), les élections au CE ne peuvent avoir lieu faute de candidats parmi les salariés, ce qui explique souvent également l'absence de CHSCT. Par ailleurs certains CHSCT peuvent avoir une activité purement formelle ou se réunir très peu souvent (même s'ils doivent légalement se réunir au moins quatre fois par an).

Au-delà des dispositions juridiques, qui comme on le voit ne suffisent pas à garantir l'existence d'une représentation $\mathrm{du}$ personnel, de quelle manière la mise en place d'un CHSCT et son dynamisme dépendent-ils de la nature des relations profession- 
nelles et des stratégies managériales spécifiques de l'établissement? On testera ici deux hypothèses, renvoyant aux effets de la pression sociale interne et de la pression organisationnelle externe.

Selon la première hypothèse (dite de «pression sociale»), l'existence d'un collectif de travail dynamique et solidaire confère aux salariés et à leurs représentants un pouvoir qui leur permet d'imposer la mise en place et le bon fonctionnement des instances représentatives du personnel en matière de santé-sécurité. Plus précisément, trois caractéristiques des relations professionnelles dans l'établissement doivent être prises en considération: "Autonomous worker representation at the workplace, trade union support and effective communication between worker representatives and their constituencies are pre-requisites for effective worker representation" (WALTERS et al., 2005, p. 118). L'autonomie de la représentation, le soutien syndical, la qualité de la communication entre les représentants et leurs mandants, chacune de ces trois variables peut faire l'objet d'une quantification grâce à l'enquête REPONSE.

Selon la seconde hypothèse («pression organisationnelle»), la mise en place d'un CHSCT est facilitée quand le management de l'entreprise, et en particulier sa gestion des ressources humaines (GRH), est professionnalisé et formalisé, ce qui est notamment le cas dans les grands groupes.

Pour tester empiriquement ces hypothèses, on a estimé un modèle Logit afin d'évaluer l'influence respective de divers facteurs sur la probabilité qu'un établissement dispose d'un CHSCT (modèle 1, tableau 1), et sur la probabilité qu'un CHSCT donné tienne davantage de réunions que le nombre minimum légal (quatre par an) (modèle 2). Ce dernier indicateur est ici supposé refléter à la fois l'intensité de l'activité des RP-SS, et la disposition du management à mener une politique efficace de prévention, deux paramètres dont on a vu qu'ils sont souvent associés dans la littérature scientifique sur le thème.

La taille de l'établissement vient bien sûr en premier au rang des déterminants de la présence d'un CHSCT. Dans le cas français, ceci reflète d'abord la législation, qui prévoit l'obligation de créer un CHSCT à partir de 50 salariés. Le secteur d'activité joue un rôle moindre: cependant les secteurs de services ont une propension plus faible, toutes choses égales par ailleurs, à mettre en place un CHSCT.

L'influence des politiques managériales apparaît clairement, confirmant l'hypothèse de pression organisationnelle. Tout d'abord, appartenir à un groupe renforce la probabilité pour un établissement d'avoir un CHSCT. C'est encore plus le cas lorsque le groupe est coté en bourse. On peut donner de ces résultats l'interprétation suivante: ces établissements suivent souvent des politiques formalisées de GRH, décidées centralement par la maison-mère, notamment en ce qui concerne le fonctionnement des institutions représentatives et la prévention des risques professionnels. De même, les responsables d'entreprises qui participent activement à des structures professionnelles extérieures (fédération de branche, club d'entrepreneurs, conseil d'administration d'une autre entreprise, etc. ) ont davantage tendance que les autres à mettre en place un CHSCT: c'est très probablement parce qu'ils ont ainsi accès à de l'information et à des références en matière d'outils de management. Cet effet de l'appartenance à des réseaux patronaux extérieurs à l'entreprise s'observe surtout au plan statistique pour les PME, car la plupart des grandes entreprises sont insérées dans de tels réseaux et mettent en œuvre des politiques de GRH formalisées.

La présence d'un CHSCT dépend donc des politiques managériales, mais elle renvoie aussi aux relations professionnelles internes à l'établissement, confirmant ainsi clairement l'hypothèse de pression sociale, qui renvoie aux mécanismes politiques (Worker Power). Chacun des trois critères évoqués par D. Walters - «représentation autonome», «soutien syndical» et «communication effective» entre les représentants et leurs mandataires - peut faire l'objet d'un indicateur issu de «REPONSE»; tous exercent une influence positive et significative sur la présence du CHSCT.

Le «soutien syndical» est garanti d'abord par la présence d'une organisation syndicale dans l'entreprise ou l'établissement. En France, les employeurs de plus de 50 salariés sont tenus de reconnaître l'existence d'une délégation syndicale quand un ou plusieurs salariés sont désignés par un syndicat pour représenter les intérêts des salariés dans l'entreprise. En pratique, $63 \%$ des établissements de 50 salariés et plus (et 38\% de l'ensemble des établissements) sont couverts par une présence syndicale (PIGNONI, 2007), même si seulement $8 \%$ des salariés sont adhérents d'une organisation syndicale - constituant ce que Loup Wolff a appelé le «paradoxe du syndicalisme français: un faible nombre d'adhérents mais des syndicats bien implantés » (WoLfF, 2008).

Sans ambigüité (y compris toutes choses égales par ailleurs, tableau 1), les CHSCT sont plus présents dans les établissements qui disposent d'une représentation syndicale. Ainsi, entre 50 et 99 salariés, $65 \%$ des établissements couverts par un délégué syndical disposent d'un CHSCT contre 44\% des établissements non couverts (COUTROT, 2007). De même, toutes choses égales d'ailleurs (tableau 1), la faiblesse du taux de syndicalisation dans l'établissement joue contre la présence d'un CHSCT.

Dans la littérature internationale «l'autonomie» des représentants par rapport à la direction est le plus souvent évaluée par leur mode de nomination (choisis par la direction, élus par le personnel, ou nommés par un syndicat) (Nichols, Walters, 
Tableau 1 : La pression sociale renforce la probabilité de présence d'un CHSCT

\begin{tabular}{|c|c|c|c|}
\hline & Fréquence & $\begin{array}{c}\text { Modèle } \mathbf{1} \\
\text { Odds-ratio } \\
\text { présence d'un CHSCT }\end{array}$ & $\begin{array}{c}\text { Modèle } 2 \\
\text { Odds-ratio } \\
<+ \text { de quatre réunions } \\
\text { de CHSCT }\end{array}$ \\
\hline $\begin{array}{l}\text { N } \\
\text { Taille de l'établissement }\end{array}$ & 2930 établissements & 2049 sur 2930 & 707 sur 2049 \\
\hline 20-49 salariés & $57 \%$ & 0,03 & NS \\
\hline 100 et plus (ref.) & $22 \%$ & - & - \\
\hline \multicolumn{4}{|l|}{ Secteur d'activité } \\
\hline Industries agro-alimentaires & $3 \%$ & 2,27 & NS \\
\hline Industries biens de consommation & $4 \%$ & 2,61 & NS \\
\hline Industrie biens d'équipement & $6 \%$ & 2,30 & NS \\
\hline Transports (réf.) & $7 \%$ & - & - \\
\hline Banques, assurances & $4 \%$ & NS & 2,46 \\
\hline Services aux entreprises & $18 \%$ & NS & NS \\
\hline Services aux particuliers & $8 \%$ & NS & NS \\
\hline Santé, action sociale & $9 \%$ & NS & 0,53 \\
\hline \multicolumn{4}{|l|}{ Ancienneté de l'établissement } \\
\hline Plus de dix ans & $60 \%$ & 1,66 & NS \\
\hline \multicolumn{4}{|l|}{ Structure capitalistique de l'entreprise } \\
\hline Appartenance à un groupe & $51 \%$ & 1,30 & 1,32 \\
\hline Nombreux conflits & $12 \%$ & 1,48 & 1,98 \\
\hline Nombreuses sanctions & $30 \%$ & NS & 0,77 \\
\hline \multicolumn{4}{|l|}{ Santé-sécurité } \\
\hline Accidents du travail répétés & $11 \%$ & $1,44^{(*)}$ & $1,32^{(*)}$ \\
\hline
\end{tabular}

Source: enquête REPONSE 2004, secteur marchand non agricole.

Note de lecture: tous les odds-ratios présentés sont significatifs au seuil de $5 \%$ (sauf ${ }^{(*)}$ : au seuil de $10 \%$ seulement, et NS : non significatif).

Signification des variables :

- «réseaux patronaux»: l'entreprise participe régulièrement à deux instances extérieures (fédération de branche, association professionnelle, club patronal, conseil d'administration extérieur au groupe);

- «management prosyndical» : le représentant de la direction estime que «les syndicats jouent un rôle irremplaçable dans la représentation des salariés»; - «nombreux conflits»: l'établissement a connu au moins deux formes distinctes de conflit au cours des trois dernières années (parmi les huit formes de conflit suivantes: pétition, manifestation, débrayage, grève perlée, grève du zèle, refus d'heures supplémentaires, grève de moins de deux jours, grève de deux jours et plus);

- «nombreuses sanctions»: la proportion de salariés sanctionnés en 2004 dépasse $6 \%$;

- «accidents du travail répétés »: l'employeur signale que l'établissement a connu des «accidents du travail répétés » au cours des trois dernières années.

On observe enfin que la présence d'un CHSCT, et le fait qu'il tienne un nombre de réunions supérieur au minimum obligatoire, est corrélée positivement, toutes choses égales par ailleurs, avec la déclaration plus fréquente par l'employeur de l'existence «d'accidents du travail répétés» au cours de l'année précédente. Ce résultat n'est pas contrôlé par la présence d'expositions professionnelles: il est cohérent avec l'hypothèse selon laquelle les CHSCT sont plus présents quand le travail est dangereux, ce que l'enquête SUMER va maintenant nous permettre de confirmer.

TASIRAN, 2007). En pratique, en France les membres du CHSCT sont en général des représentants syndicaux quand l'entreprise dispose d'une présence syndicale. Mais l'enquête REPONSE permet d'aller plus loin que le mode de nomination ou l'appartenance à un syndicat: on dispose en effet d'indicateurs sur les conflits du travail, qui reflètent la capacité des représentants et des salariés à agir collectivement de façon autonome par rapport à la direction pour défendre leurs intérêts dans l'entreprise (CÉZARD, MALAN, ZoUARY, 1995). À cette aune, la présence d'un CHSCT est fortement associée à l'existence de collectifs autonomes : ainsi, parmi les établissements qui disposent d'une représentation syndicale, on trouve un CHSCT dans seulement $48 \%$ de ceux qui n'ont connu aucune forme de 
conflit collectif du travail au cours des trois années précédant l'enquête (2002 à 2004), contre $64 \%$ quand il y a eu une forme de conflit et $74 \%$ quand l'établissement en a connu plusieurs (1). Ce résultat est d'autant plus probant qu'une attitude conciliante du management vis-à-vis de la représentation des salariés devrait rendre moins probable la survenue de conflits et plus probable la création d'un CHSCT.

Enfin l'effectivité de la communication entre les salariés et leurs représentants peut être évaluée à partir des réponses des salariés eux-mêmes. $63 \%$ de ceux-ci s'estiment en accord avec l'opinion selon laquelle «les représentants du personnel traduisent bien les aspirations des salariés », et $33 \%$ signalent qu'ils utilisent en priorité le canal des représentants du personnel pour exprimer certains de leurs griefs (2). Cette qualité de la communication est en effet liée à la présence d'un CHSCT: celle-ci fait passer les pourcentages précédents à $65 \%$ et $37 \%$, et ces différences, bien que faibles en apparence, sont statistiquement très significatives (3).

La majorité (57\%) des CHSCT s'est réunie quatre fois en 2004 comme le prévoit la réglementation. Un CHSCT sur cinq n'a toutefois pas tenu le nombre légal de réunions, alors qu'un autre CHSCT sur cinq l'a dépassé. On fait ici l'hypothèse que si certains CHSCT ont une activité largement formelle et tiennent les quatre réunions obligatoires par an uniquement pour respecter la réglementation, à l'inverse le fait de tenir plus de quatre réunions est le signe d'une activité réelle, déterminée par d'autres objectifs que le simple respect d'une obligation juridique. La conflictualité interne à l'établissement renforce nettement la probabilité que le CHSCT ait une activité réelle: quand il n'y a pas eu de conflits sur les trois années précédant l'enquête, seulement $5 \%$ des CHSCT se sont réunis plus de quatre fois, contre $25 \%$ dans les établissements qui ont connu plusieurs types de conflits. L'ancienneté de l'établissement, l'insertion de la direction dans des instances professionnelles extérieures à l'entreprise, une attitude favorable au syndicalisme, sont des facteurs qui accroissent également les chances que

(1) $C f$. tableau 1 pour la liste des types de conflits retenus dans l'enquête REPONSE.

(2) Plus exactement, $33 \%$ des salariés répondent «s'adresser en priorité aux représentants du personnel pour essayer de trouver une solution" pour au moins un des quatre types de problèmes évoqués dans le questionnaire (conditions de travail, absence de promotion, mésentente avec un supérieur, procédure de licenciement).

(3) C'est ce que montre un modèle Logit (non reproduit) calculé sur les seuls établissements pour lesquels on dispose de réponses de salariés, et expliquant la présence de CHSCT par les mêmes variables que le modèle du tableau 1 , auxquelles on a ajouté les deux indicateurs de qualité de la communication entre salariés et représentants issus du questionnaire salariés. Les odd-ratios des variables en question sont de 1,35 (pour la variable «le salarié s'adresse en priorité aux représentants du personnel») et 1,19 (pour la variable «le salarié considère que les représentants traduisent bien les aspirations des salariés»), significatifs au seuil de $1 \%$. le CHSCT se réunisse plus souvent que le minimum légal (tableau 1).

\section{Risques professionnels et mise en place d'un CHSCT}

Concernant les liens entre expositions aux risques professionnels et présence de CHSCT, plusieurs hypothèses peuvent être avancées. D'une part la présence de risques devrait favoriser la mise en place d'un CHSCT: d'abord parce que l'importance des expositions peut inciter l'entreprise au respect du Code du travail, par souci de la santé des salariés ou par crainte des sanctions et pénalités financières que pourraient prendre l'inspection du travail ou les assureurs (les caisses primaires d'assurancemaladie) (effet «réglementation»)(4); mais aussi à cause d'un effet cognitif (Worker et Management Knowledge), l'importance des expositions pouvant favoriser la prise de conscience des dangers par les acteurs, et donc la création du CHSCT.

En sens inverse une politique active de prévention, favorisée par l'existence d'un CHSCT, peut aboutir à l'atténuation ou l'élimination des risques à la source (effet «prévention primaire»). Nos données sont en coupe instantanée et ne permettent pas de distinguer la période d'émergence du CHSCT et son impact ultérieur sur l'évolution des expositions. Le lien statistique éventuellement observé entre expositions et présence d'un CHSCT rendra donc compte de la résultante d'effets contradictoires; une absence de lien statistique entre CHSCT et expositions n'indiquerait d'ailleurs pas nécessairement une absence de lien causal, car il se peut que les fortes expositions aient favorisé la création d'un CHSCT, et que l'action de ce dernier ait ensuite permis de réduire les expositions.

On mesure donc ici, en coupe instantanée, la corrélation entre présence du CHSCT et expositions professionnelles, en contrôlant l'estimation par un grand nombre de variables de contexte (taille, secteur, composition de la main-d'œuvre, etc. ) (tableau 2). La présence d'un CHSCT est une caractéristique de l'établissement, alors que les expositions professionnelles caractérisent un poste de travail particulier, pas nécessairement représentatif de la situation moyenne de l'établissement: il y a donc un risque de «bruit» statistique qui pourrait induire une sousestimation de l'intensité des liens entre expositions et CHSCT. Les évaluations ci-dessous doivent donc être considérées comme des bornes inférieures de l'intensité des liens mesurés.

(4) Ainsi l'inspection du travail peut imposer la création d'un CHSCT dans un établissement de moins de cinquante salariés «en raison notamment de la nature des travaux, de l'agencement ou de l'équipement des locaux». 
Pour synthétiser la très grande variété des expositions professionnelles décrites dans le questionnaire de l'enquête SUMER 2003, douze indicateurs synthétiques d'expositions aux différents risques recensés ont été construits:

- une exposition à au moins un produit chimique (5): avec une intensité qui n'est pas déclarée « très faible » par le médecin du travail (fréquence $=21 \%$ );

- une exposition aux agents biologiques (quelle qu'en soit la durée) (fréquence 15\%);

- une exposition à des radiations ou au rayonnement (quelle qu'en soit la durée) (fréquence 4\%);

- une exposition à de fortes contraintes de rythme de travail : le rythme de travail du salarié est imposé par au moins trois contraintes sur les neuf recensées par l'enquête (6) (fréquence $30 \%$ );

- une exposition à des efforts physiques importants (7) (fréquence 24\%);

- une exposition forte au travail sur écran: le salarié travaille plus de 20 heures par semaine sur un système informatique (fréquence $21 \%$ );

- une exposition au travail de nuit: plus de vingt nuits par an (fréquence $12 \%$ )

- une exposition au travail posté $(2 \times 8,3 \times 8$, etc. $)$ (fréquence $13 \%$ );

- des horaires imprévisibles: le salarié ne connaît pas ses horaires de la semaine prochaine (fréquence $13 \%$ );

- une durée longue du travail : 45 heures ou plus lors la dernière semaine travaillée (fréquence 14\%);

- un manque de marges de manœuvre: en cas d'incident le salarié fait généralement appel à d'autres (fréquence $22 \%$ );

- un soutien social insuffisant: le salarié déclare ne pas avoir la possibilité de coopérer pour faire correctement son travail (fréquence $7 \%$ ).

Les résultats du modèle (tableau 2) indiquent que certaines expositions sont associées à une présence

(5) Le médecin du travail qui remplit le volet «Agents chimiques » du questionnaire SUMER est amené, pour chacun des quatre-vingts produits chimiques recensés, à préciser l'intensité de l'exposition («estimée très faible», «mesurée très faible», «estimée faible», «mesurée faible», «estimée forte», «mesurée forte», «estimée très forte», «mesurée très forte»). On a retenu ici les expositions dont l'intensité n'était pas estimée ou mesurée «très faible», soit un peu plus de la moitié des expositions.

(6) Les neuf contraintes de rythme sont: déplacement automatique d'une pièce ou cadence automatique d'une machine; autres contraintes techniques; dépendance immédiate du rythme de travail des collègues; norme de production ou délai à respecter en une heure au plus; norme ou délai à respecter en une journée au plus; demande extérieure obligeant à une réponse immédiate; demande extérieure n'obligeant pas à une réponse immédiate; contrôle ou surveillance permanents d'un chef; contrôle ou suivi informatisé.

(7) Le salarié est exposé à au moins deux des six efforts suivants : position debout plus de 20 heures par semaine, déplacement à pied plus de 20 heures par semaine, répétition d'un même geste plus de 20 heures par semaine, travail à genoux plus de 2 heures par semaine, travail bras en l'air plus de 2 heures par semaine, travail en torsion ou accroupi plus de 2 heures par semaine.
Tableau 2: Certaines expositions professionnelles renforcent la probabilité de présence d'un CHSCT

\begin{tabular}{|c|c|c|}
\hline & $\begin{array}{c}\text { \% de } \\
\text { CHSCT }\end{array}$ & $\begin{array}{c}\text { Odds-ratio } \\
\text { (modèle Logit) }\end{array}$ \\
\hline Tous établissements & 50 & \\
\hline \multicolumn{3}{|l|}{$\begin{array}{l}\text { Caractéristiques de } \\
\text { l'établissement }\end{array}$} \\
\hline $\begin{array}{l}\text { Possède un service autonome } \\
\text { de médecine du travail }\end{array}$ & 94 & 3,94 \\
\hline Norme ISO & 78 & 2,68 \\
\hline Passé aux 35 heures & 59 & 4,41 \\
\hline \multicolumn{3}{|l|}{ Taille } \\
\hline$<20$ salariés & 10 & 0,04 \\
\hline 20-49 salariés & 24 & 0,06 \\
\hline 50-499 salariés & 81 & Ref. \\
\hline 500 salariés et plus & 97 & 5,60 \\
\hline \multicolumn{3}{|l|}{ Secteur } \\
\hline BTP & 30 & 0,68 \\
\hline Services aux particuliers & 29 & 0,68 \\
\hline \multicolumn{3}{|l|}{ Caractéristiques du salarié } \\
\hline Homme & 52 & 1,14 \\
\hline Ancienneté $<1$ an & 27 & 0,73 \\
\hline CDD & 34 & 0,81 \\
\hline Intérimaire & 25 & 0,12 \\
\hline Fonctionnaire & 89 & 0,60 \\
\hline Temps partiel & 36 & 0,86 \\
\hline \multicolumn{3}{|l|}{$\begin{array}{l}\text { Expositions professionnelles } \\
\text { liées au poste de travail }\end{array}$} \\
\hline $\begin{array}{l}\text { Durée hebdomadaire longue } \\
(>=45 \text { heures })\end{array}$ & 51 & NS \\
\hline Travail posté & 79 & 1,46 \\
\hline $\begin{array}{l}\text { Travail de nuit (plus de } 20 \\
\text { nuits/an) }\end{array}$ & 65 & 1,13 \\
\hline Horaires imprévisibles & 43 & 0,88 \\
\hline $\begin{array}{l}\text { Travail sur écran (plus } \\
20 \text { heures/semaine) }\end{array}$ & 58 & 1,20 \\
\hline Fortes contraintes physiques & 46 & NS \\
\hline Bruit & 54 & NS \\
\hline Agents biologiques & 56 & NS \\
\hline $\begin{array}{l}\text { Agents chimiques (exposition } \\
\text { non négligeable) }\end{array}$ & 47 & 0,89 \\
\hline Radiations, rayonnement & 66 & 1,27 \\
\hline $\begin{array}{l}\text { Fortes contraintes de rythme } \\
\text { de travail }\end{array}$ & 61 & 1,30 \\
\hline Faibles marges de manœuvre & 48 & NS \\
\hline Faible soutien social & 45 & NS \\
\hline
\end{tabular}

Source: enquête SUMER 2003.

Note de lecture : $50 \%$ des salariés signalent un CHSCT dans leur établissement, mais $94 \%$ dans les établissements disposant d'un service autonome de médecine du travail; toutes choses égales par ailleurs, disposer d'un service autonome multiplie par 3,94 la probabilité d'existence d'un CHSCT. Les odds-ratios ici indiqués sont significatifs au seuil de $1 \%$ (sauf NS: non significatif). 
Tableau 3 : Les CHSCT sont associés à une meilleure qualité de la prévention et de l'information

\begin{tabular}{|c|c|c|c|c|}
\hline & Avec CHSCT & Sans CHSCT & Ensemble & Odds-ratios \\
\hline \multicolumn{5}{|c|}{ \% de salariés exposés disposant d'une protection individuelle contre: } \\
\hline - le bruit & $73 \%$ & $60 \%$ & $67 \%$ & $1,18^{(*)}$ \\
\hline - les rayonnements & $47 \%$ & $44 \%$ & $45 \%$ & NS \\
\hline - les agents biologiques & $59 \%$ & $35 \%$ & $49 \%$ & 1,30 \\
\hline - les agents chimiques & $67 \%$ & $57 \%$ & $62 \%$ & 1,19 \\
\hline \multicolumn{5}{|l|}{ Jugement négatif du médecin sur le: } \\
\hline -risque organisationnel $(1)$ & $20 \%$ & $17 \%$ & $18 \%$ & 1,16 \\
\hline -risque physique (2) & $31 \%$ & $32 \%$ & $31 \%$ & 1,10 \\
\hline - risque chimique (3) & $25 \%$ & $35 \%$ & $30 \%$ & 0,81 \\
\hline -risque biologique (4) & $12 \%$ & $22 \%$ & $17 \%$ & 0,83 \\
\hline $\begin{array}{l}\text { Le salarié a eu un accident avec arrêt de travail } \\
\text { au cours des douze derniers mois 㛽- }\end{array}$ & $4,1 \%$ & $4,6 \%$ & $4,4 \%$ & NS \\
\hline Le salarié estime que son travail est fatigant & $29 \%$ & $27 \%$ & $28 \%$ & NS \\
\hline Le salarié estime que son travail est stressant 浨 & $38 \%$ & $32 \%$ & $35 \%$ & NS \\
\hline Le salarié estime que son travail est mauvais pour sa santé & $30 \%$ & $24 \%$ & $27 \%$ & 1,13 \\
\hline Le salarié estime que sa santé est médiocre 姲 & $21 \%$ & $18 \%$ & $19 \%$ & NS \\
\hline
\end{tabular}

Source: enquête SUMER 2003 (autoquestionnaire).

(1) Champ: tous salariés secteur marchand.

(2) Champ: salariés exposés à une contrainte physique (soit $77 \%$ des salariés).

(3) Champ: salariés exposés à un agent biologique (soit $15 \%$ ).

(4) Champ: salariés exposés à un agent chimique (soit 38\%).

Les odds-ratios indiqués sont significatifs au seuil de $1 \%$ ( $\operatorname{sauf}(*)$ : au seuil de $10 \%$, ou NS: non significatif). Ils reflètent l'influence de la présence d'un CHSCT sur la probabilité d'occurrence de l'événement (par exemple avoir eu un accident du travail), dans un modèle «Logit» où les variables de contrôle sont les caractéristiques de l'établissement, du salarié et du poste de travail (expositions professionnelles) ( $c f$. tableau 2). A titre d'exception les modèles concernant les protections individuelles n'incluent pas les expositions professionnelles comme variables de contrôle, puisqu'ils sont estimés sur la seule sous-population exposée au risque (qui peut seule bénéficier de protections).

nettement plus fréquente d'un CHSCT: le travail posté et le travail de nuit, le travail sur écran, les radiations et rayonnements, un cumul de contraintes de rythme de travail. En revanche les CHSCT sont un peu moins nombreux pour les postes exposés au risque chimique et aux horaires imprévisibles. Globalement, c'est l'effet «réglementation», conjointement aux effets de nature cognitive, qui semble dominer : les directions tendent d'autant plus à mettre en place des CHSCT que les risques professionnels sont importants dans leur établissement, $\mathrm{du}$ fait des caractéristiques techniques et organisationnelles de leur activité. Cependant on trouve des traces d'un effet «élimination à la source» concernant les expositions chimiques: c'est du moins ce que suggère le lien négatif entre risque chimique et présence de CHSCT (on aura d'ailleurs ci-dessous confirmation de cette relative efficacité).

Au-delà des expositions professionnelles, certaines caractéristiques de l'établissement renforcent considérablement la probabilité de présence d'un CHSCT: outre les grands établissements, ceux qui ont réduit la durée du travail à 35 heures, qui ont adopté une norme ISO ou qui disposent un service autonome de médecine du travail, sont beaucoup plus nombreux à avoir un CHSCT. Ces indicateurs reflètent peut-être une propension de la direction de l'établissement à se conformer aux normes sociales (35 heures), organisationnelles (ISO) ou sanitaires (service autonome), qui expliquerait aussi la conformité à la réglementation du travail. Il pourrait ici encore s'agir d'un « effet management». Pour ce qui concerne des relations sociales dans l'entreprise, la «pression sociale» interne, l'enquête SUMER ne fournit pas d'informations.

Notons également que le fait d'être une femme, d'avoir un contrat précaire (CDD ou intérim) ou à temps partiel, implique une probabilité plus faible d'être couvert par un CHSCT, en contrôlant par la présence des expositions. Ces résultats sont d'ailleurs confirmés par l'enquête Conditions de travail de 2005 (СоuтRот, 2008): les femmes et les précaires - dont la majorité sont des femmes pour les CDD et des hommes pour les intérimaires - bénéficient systématiquement moins souvent des diverses mesures de protection de la santé au travail, même à conditions de travail identiques. La capacité d'influencer les pratiques des employeurs (Worker Power) semble donc plus réduite pour ces catégories de salariés.

\section{Chsct et qualité de la prévention}

On cherche ici à évaluer la corrélation, toutes choses égales par ailleurs, entre la présence d'un CHSCT et la qualité de la prévention des risques. Les variables de contrôle sont à nouveau les caractéristiques de l'établissement, du salarié et du poste de travail, y compris les expositions elles-mêmes. 
L'enquête SUMER 2003 fournit deux types d'informations pour évaluer la qualité de la prévention sur les postes de travail. D'une part, pour les risques liés au bruit, aux rayonnements, aux agents chimiques ou aux agents biologiques, à chaque fois qu'une exposition est identifiée dans le questionnaire, le médecin doit indiquer si le salarié dispose d'une protection individuelle (auditive dans le cas du bruit, cutanée, respiratoire ou oculaire dans le cas des autres risques). La proportion de salariés disposant d'une protection individuelle varie de $45 \%$ pour les rayonnements à $67 \%$ pour le bruit (tableau 3) (8).

D'autre part, pour évaluer la qualité de la prévention, on met à profit une caractéristique unique de l'enquête SUMER, l'expertise professionnelle du médecin du travail enquêteur, à qui est demandé une appréciation synthétique, compte tenu des expositions et des mesures de prévention existantes, sur le «risque de pathologie» associé au poste de travail. En pratique, le médecin doit attribuer une note de 1 («très mauvais ») à 4 («très bon ») concernant la «qualité du poste et/ou de l'environnement de travail» dans quatre domaines: «l'organisation du travail», «la prévention des expositions à des contraintes physiques», «la prévention des expositions aux agents biologiques», «la prévention des expositions aux agents chimiques ». Pour un nombre significatif de postes (de $17 \%$ pour le risque biologique à $31 \%$ pour les contraintes physiques), les médecins du travail jugent la qualité de la prévention «mauvaise» ou «très mauvaise» (tableau 3 ).

Bien sûr, aucun de ces indicateurs ne constitue à lui seul une évaluation totalement satisfaisante de la qualité de la prévention. Ainsi la mise à disposition d'équipements de protection individuelle peut être un substitut inadéquat à des politiques d'élimination à la source des expositions ou de protection collective; ces équipements peuvent en outre être inadaptés et en pratique peu utilisés par les travailleurs (SIMARD, MARCHAND, 1999), l'enquête ne disant rien sur ce dernier point. L'opinion du médecin peut également être biaisée, du fait qu'il est pour partie responsable de la qualité de la prévention des risques des postes de travail qu'il surveille, du fait aussi de sa dépendance vis-à-vis de l'employeur: on observe par exemple qu'à expositions identiques, les médecins du travail de services autonomes (donc employés par la grande entreprise où ils travaillent) sont plus optimistes sur la qualité

(8) Il s'agit ici de la proportion de salariés exposés à un risque (par exemple chimique) disposant d'au moins une protection individuelle. du poste de travail que les médecins des services interenterprises (9).

On fera néanmoins l'hypothèse qu'à expositions égales, une entreprise qui met des protections à disposition de ses salariés déploie une politique de prévention plus active qu'une autre qui ne le fait pas. De même les appréciations des médecins du travail sont peut-être biaisées, mais ne sont pas incohérentes avec celles des salariés (recueillies indépendamment dans l'autoquestionnaire): ainsi parmi les salariés dont le médecin du travail estime que le poste de travail est de mauvaise qualité au plan organisationnel, $43 \%$ jugent que leur travail est «plutôt mauvais pour leur santé», contre $23 \%$ de ceux dont le poste n'est pas jugé de mauvaise qualité par le médecin.

Mais ce qui importe surtout est la concordance des résultats selon les indicateurs étudiés. En effet, que l'on considère l'existence de protections individuelles ou le jugement synthétique du médecin, les conclusions sont les mêmes: la présence d'un CHSCT dans l'établissement est clairement associée avec une meilleure qualité de la prévention contre les risques chimiques et biologiques. Ainsi, pour les salariés exposés à un agent chimique, $67 \%$ disposent d'une protection individuelle quand il y a un CHSCT, contre $57 \%$ en l'absence du CHSCT; toutes choses égales par ailleurs, la présence d'un CHSCT accroît de 19\% la probabilité qu'il dispose d'une protection individuelle. De même, elle réduit de $19 \%$ la probabilité que le médecin juge mauvaise la qualité du poste de travail. Ces chiffres sont respectivement de $+30 \%$ et $-17 \%$ pour le risque biologique. Ces résultats sont statistiquement très significatifs (au seuil de 1\%). Signe supplémentaire de leur robustesse, ils dépendent très peu du nombre de variables de contrôle qu'on introduit dans les modèles économétriques (tableau 4).

Concernant les risques liés au bruit, les résultats vont dans le même sens : en présence de CHSCT, $73 \%$ des salariés disposent d'une protection contre $60 \%$ en l'absence de CHSCT. Toutefois le lien est moins assuré toutes choses égales par ailleurs: l'odds-ratio $(1,18)$ n'est significatif qu'au seuil de $10 \%$.

Le lien positif entre la présence d'un CHSCT et la qualité des politiques de prévention est confirmé par les données de l'enquête Conditions de travail

(9) Les médecins de services autonomes (soit 20\% des médecins) ont une probabilité $10 \%$ inférieure (par rapport aux médecins de services interentreprises) d'estimer que le poste de travail est de mauvaise qualité au plan des expositions physiques $(20 \%$ pour les contraintes organisationnelles) (résultats de modèles Logit ici non présentés, où les variables de contrôle incluent les expositions professionnelles). Ce relatif optimisme ne reflète peut-être pas qu'une perception biaisée, dans la mesure où les entreprises disposant de services autonomes ont peut-être une politique de prévention plus efficace, mais l'enquête SUMER ne permet pas d'en juger. 
Tableau 4: Test de robustesse des modèles explicatifs de la qualité de la prévention

\begin{tabular}{|c|c|c|c|}
\hline $\begin{array}{l}\text { Odds-ratio de la variable CHSCT dans un modèle multivarié Logit } \\
\text { expliquant la «mauvaise qualité du poste» du point de vue... }\end{array}$ & Modèle 1 & Modèle 2 & Modèle 3 \\
\hline de l'organisation du travail ${ }^{(1)}$ & $1,17(* * *)$ & $1,17(* * *)$ & $1,16(* * *)$ \\
\hline de la prévention des expositions à des contraintes physiques ${ }^{(2)}$ & 1,01 & $1,11(* * *)$ & $1,10(* * *)$ \\
\hline de la prévention des expositions aux agents biologiques ${ }^{(3)}$ & $0,82(*)$ & 0,84 & $0,81(*)$ \\
\hline de la prévention des expositions aux agents chimiques ${ }^{(4)}$ & $0,77(* * *)$ & $0,82(* * *)$ & $0,83(* * *)$ \\
\hline
\end{tabular}

Source: enquête SUMER 2003.

(1) Champ : tous salariés.

(2) Champ: salariés exposés à une contrainte physique (soit $77 \%$ des salariés).

(3) Champ: salariés exposés à un agent biologique (soit $15 \%$ ).

(4) Champ: salariés exposés à un agent chimique (soit $38 \%$ ).

Modèle 1: variables de contrôle = seulement les caractéristiques de l'établissement.

Modèle 2: idem 1 plus caractéristiques du salarié.

Modèle 3 : idem 2 plus expositions aux risques professionnels.

Note de lecture : *** (respectivement *) indique un odds-ratio significatif au seuil de $1 \%$ (respectivement $10 \%)$.

Tableau 5: Les CHSCT sont associés à une meilleure qualité de la prévention et de l'information

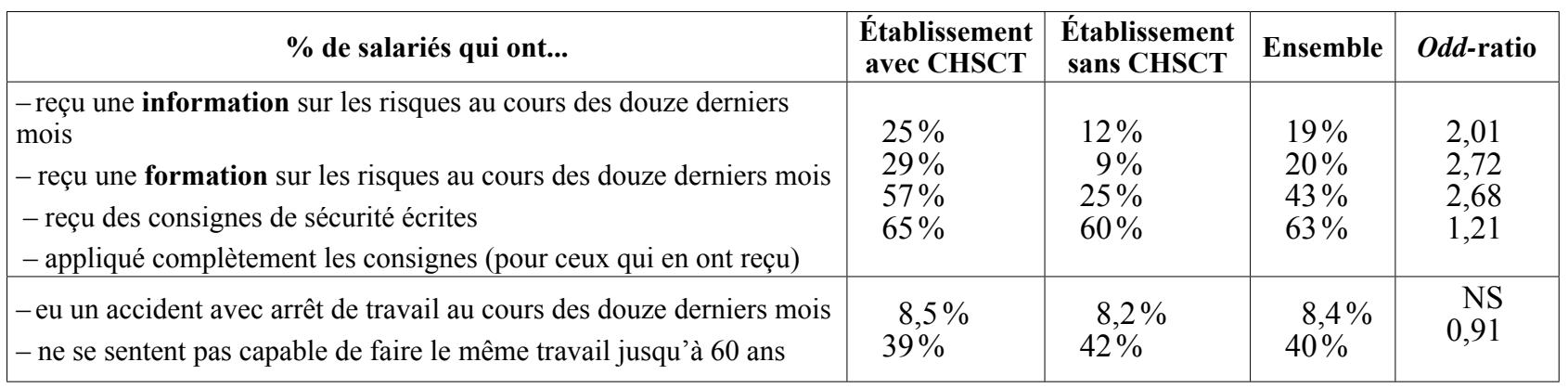

Source: enquête Conditions de travail 2005, Insee-Dares.

Champ: tous salariés, tous secteurs.

Lecture: dans les établissements qui disposent d'un CHSCT $25 \%$ des salariés ont reçu une information sur les risques au cours des douze derniers mois, contre $12 \%$ dans les établissements sans CHSCT.

Les odds-ratios indiqués sont significatifs au seuil de $1 \%$ (sauf NS : non significatif). Ils reflètent l'influence de la présence d'un CHSCT sur la probabilité d'occurrence de l'événement (par exemple avoir reçu une information sur les risques), dans un modèle Logit où les variables de contrôle sont les caractéristiques de l'établissement, du salarié et du poste de travail (expositions professionnelles).

(Coutrot, 2008). Toutes choses égales par ailleurs (taille et secteur de l'établissement, caractéristiques du salarié et de son poste de travail), la présence d'un CHSCT fait plus que doubler la probabilité qu'un salarié ait reçu une formation ou une information sur les risques professionnels au cours des douze derniers mois. Elle multiplie également par un facteur supérieur à 2 la probabilité que le salarié dispose de consignes de sécurité écrite (tableau 5bis). Enfin, quand il y a un CHSCT, la probabilité que le salarié déclare appliquer totalement les consignes écrites dont il dispose est accrue de $20 \%$.

\section{ChSCT et connaissance des risques}

En revanche la présence d'un CHSCT a pour effet d'accroître significativement la probabilité que le médecin juge le poste de travail de mauvaise qualité sur le plan de l'organisation du travail $(+16 \%)$ et des risques physiques $(+10 \%)$. De même elle accroît de $13 \%$ la probabilité que le salarié juge que son travail a un effet négatif sur sa santé (tableau 3). Pourtant, toutes choses égales par ailleurs, elle n'a pas de lien significatif avec les indicateurs de santé disponibles dans l'enquête SUMER : la probabilité d'avoir subi un accident du travail au cours des douze derniers mois, celle de se déclarer en médiocre santé(10), ou de déclarer son travail «fatigant» ou «stressant». Toutefois, quelle que soit la définition de la santé adoptée, qu'on l'envisage comme l'absence de maladies ou (selon l'Organisation mondiale de la santé) comme un «état de complet bien-être physique, mental

(10) On considère ici comme médiocre la santé des personnes qui attribuent à leur état de santé une note inférieure ou égale à 6 (sur une échelle de 1 - très mauvaise - à 10 - très bonne), soit $19 \%$ des salariés. 
Tableau 6: Analyse économétrique de l’opinion du médecin sur la qualité de la prévention contre les différents risques (modèles Logit)

Odds-ratios

\begin{tabular}{|c|c|c|c|c|}
\hline Mauvaise prévention des risques ... & Physiques & Organisationnels & Chimiques & Biologiques \\
\hline \multicolumn{5}{|l|}{ Caractéristiques de l'établissement } \\
\hline Possède un service autonome de médecine du travail & 0,83 & 0,74 & 0,76 & NS \\
\hline Norme ISO & 0,78 & 0,80 & 0,85 & 0,82 \\
\hline Passé aux 35 heures & 0,88 & 0,89 & 0,90 & NS \\
\hline Possède un CHSCT & 1,10 & 1,16 & 0,83 & $0,81^{(*)}$ \\
\hline$<20$ salariés & 0,80 & 0,76 & NS & 1,33 \\
\hline $20-49$ & NS & NS & NS & 1,32 \\
\hline $50-499$ & Ref. & Ref. & Ref. & Ref. \\
\hline 500 et plus & NS & NS & 0,72 & 0,78 \\
\hline Secteur & NS & & NS & NS \\
\hline BTP & & 0,70 & & \\
\hline \multicolumn{5}{|l|}{ Caractéristiques du salarié } \\
\hline Homme & 0,72 & 0,84 & 1,17 & 1,19 \\
\hline Ancienneté $<1$ an & NS & NS & NS & NS \\
\hline CDD & NS & NS & NS & NS \\
\hline Intérimaire & NS & NS & NS & NS \\
\hline Étranger non communautaire & 1,43 & 1,31 & NS & NS \\
\hline Temps partiel & NS & NS & 0,87 & NS \\
\hline \multicolumn{5}{|l|}{ Expositions professionnelles } \\
\hline Durée hebdomadaire longue ( $>=45$ heures) & NS & 1,81 & NS & NS \\
\hline Travail posté & 1,17 & 1,10 & NS & NS \\
\hline Travail de nuit (plus de 20 nuits/an) & NS & 1,25 & 0,79 & NS \\
\hline Horaires imprévisibles & 1,15 & 1,62 & NS & NS \\
\hline Travail sur écran (plus de 20 heures/semaine) & 0,77 & 1,12 & NS & NS \\
\hline Fortes contraintes physiques & 1,30 & 1,16 & 1,17 & NS \\
\hline Bruit élevé (> $85 \mathrm{db})$ & 1,44 & 1,21 & 1,45 & 1,36 \\
\hline Agents biologiques & 1,27 & 1,17 & 0,87 & $(*)$ \\
\hline Agents chimiques (exposition non négligeable) & 1,38 & 1,11 & 2,10 & 1,28 \\
\hline Radiations, rayonnement & 1,18 & 1,19 & 1,14 & NS \\
\hline Fortes contraintes de rythme de travail & 1,33 & 1,67 & 1,13 & 1,20 \\
\hline Faibles marges de manœuvre & NS & 1,11 & NS & NS \\
\hline Faible soutien social & 1,66 & 2,64 & 1,29 & 1,28 \\
\hline $\mathrm{N}$ & $\begin{array}{l}38281 \\
(77 \%) \\
\end{array}$ & $49855(100 \%)$ & $18904(38 \%)$ & $7692(15 \%)$ \\
\hline Champ du modèle & $\begin{array}{l}\text { Au moins } \\
\text { un risque } \\
\text { physique }\end{array}$ & Champ complet & $\begin{array}{l}\text { Au moins une } \\
\text { exposition } \\
\text { chimique }\end{array}$ & $\begin{array}{l}\text { Au moins une } \\
\text { exposition } \\
\text { biologique }\end{array}$ \\
\hline
\end{tabular}

Source: enquête SUMER 2003.

Note de lecture: à caractéristiques identiques de l'établissement, du salarié et du poste de travail, un médecin du travail enquêteur appartenant à un service autonome de santé au travail a une probabilité inférieure de $17 \%$ (odds-ratio $=0,83$ ) de signaler un risque de pathologie dû aux risques physiques, relativement à un médecin d'un service interentreprises. À l'inverse, quand il y a un CHSCT dans l'établissement, la probabilité de signaler un risque de pathologie lié aux contraintes physiques est accrue de $10 \%$. Le secteur d'activité n'a pas d'influence significative, sauf le BTP pour les risques organisationnels. 
et social» auquel le travail devrait contribuer, les indicateurs ici disponibles ne permettent pas d'en rendre compte avec une précision satisfaisante.

Il n'en demeure pas moins que médecins du travail et salariés apparaissent plus inquiets des conséquences du travail sur la santé en présence d'un CHSCT. Comment l'expliquer? On l'a $\mathrm{vu}$, les CHSCT sont davantage présents dans les établissements comportant des expositions importantes aux risques physiques et organisationnels ( $c f$. tableau 2). Toutefois, les expositions figurent parmi les variables de contrôle (tableau 6), et jouent en effet fortement sur 1'inquiétude du médecin: ainsi les horaires imprévisibles (le fait de «ne pas connaître ses horaires de la semaine prochaine») accroissent de $62 \%$ la probabilité que le médecin juge le poste de mauvaise qualité sur le plan organisationnel. Ce n'est donc pas seulement du fait de ces expositions que médecins et salariés sont plus inquiets. La présence d'un CHSCT aurait-elle pour effet paradoxal de dégrader la qualité de la prévention contre les risques physiques et organisationnels? Un tel résultat semble peu plausible au plan théorique, et on n'observe d'ailleurs aucun lien entre la présence du CHSCT et les accidents du travail ou la mauvaise santé perçue. Les salariés couverts par un CHSCT sont plus inquiets de l'effet de leur travail sur leur santé, mais ils ne sont pas (toutes choses égales par ailleurs) en moins bonne santé que les autres salariés. C'est donc que le CHSCT n'augmente pas les risques eux-mêmes mais bien la connaissance qu'en ont les acteurs (Worker Knowledge). L'activité du CHSCT, les échanges lors de ses réunions, les enquêtes qu'il peut diligenter, donnent une visibilité à des risques qui pourraient autrement passer inaperçus.

À la différence du risque chimique ou biologique, il n'existe pas pour les risques physique et organisationnel d'autre dispositif de prévention que l'élimination (ou l'atténuation) à la source: on ne peut pas interposer entre la nuisance et le salarié un dispositif de protection pour atténuer le risque. Le questionnaire SUMER ne comporte donc pas de questions sur les protections contre le risque physique ou organisationnel, ce qui permettrait (comme pour les risques chimique et biologique) d'évaluer plus objectivement l'impact du CHSCT sur la prévention. On peut seulement rappeler que les CHSCT sont plus présents dans des établissements où les risques organisationnels sont importants (tableau 2): les résultats ici présentés semblent indiquer que les CHSCT ne parviennent pas à réduire significativement les risques physiques, organisationnels et psychosociaux, alors que leur action dans le domaine des risques chimiques et biologiques apparaît efficace.

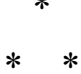

Les résultats de l'étude, obtenus en utilisant plusieurs types d'indicateurs et plusieurs sources statistiques, sont cohérents avec les hypothèses théoriques retenues. La présence et l'activité des représentants des salariés en matière d'hygiène, de sécurité et de conditions de travail (les CHSCT) reflètent la nature des relations professionnelles dans l'entreprise, et en particulier la capacité des collectifs de travail à engager des actions collectives de manière autonome par rapport à la direction (Worker Power). La présence d'un CHSCT est clairement associée à une meilleure qualité des politiques de prévention dans les entreprises et à une plus grande sensibilisation des acteurs aux risques du travail (Worker Knowledge). Ces résultats sont d'autant plus probants qu'ils s'appuient sur des sources statistiques variées, constituées avec de grands échantillons représentatifs au plan national, et qui ont permis de construire pour cette première étude sur le cas français des indicateurs rarement ou jamais pris en compte dans la littérature internationale sur le sujet. Ainsi, la mise à disposition d'équipements de protection, l'offre de formations à la santé-sécurité, l'existence de consignes de sécurité écrites, leur respect par les salariés, le jugement des médecins du travail sur la qualité de la prévention, tous ces indicateurs sont significativement plus favorables dans les établissements dotés de CHSCT. En outre, nous avons pu montrer que cette association entre représentation du personnel et qualité de la prévention dépend des risques considérés: elle est nette pour les risques chimiques et biologiques, mais n'apparaît pas pour les risques physiques et organisationnels.

Pour autant, corrélation n'est pas causalité, et l'absence de données longitudinales ne permet pas d'attribuer aux seuls CHSCT ces résultats; il n'est pas exclu que d'autres variables non observées, comme la qualité du management ou son degré d'implication dans les politiques de prévention, contribuent à expliquer à la fois la présence d'un CHSCT et la meilleure qualité de la prévention.

En revanche il n'a pas ici été possible d'établir de lien direct entre la présence d'un CHSCT et l'état de santé des salariés, peut-être en partie du fait de la nature trop grossière des indicateurs de santé disponibles dans les enquêtes mobilisées. En particulier la présence d'un CHSCT n'a pas de lien apparent avec le taux d'accidents du travail signalés par les salariés; on observe même dans l'enquête REPONSE une corrélation positive entre la présence de CHSCT et l'occurrence d'accidents du travail répétés signalée par l'employeur. Des résultats similaires se rencontrent dans la littérature scientifique internationale (Nichols, Walters, TASIRAN, 2007), ce qui peut s'expliquer notamment par le fait (ici 
documenté) que les CHSCT sont davantage présents quand les risques professionnels sont importants: en coupe instantanée il est donc impossible d'observer l'effet éventuellement favorable de l'action du CHSCT sur le taux d'accident. Dans l'enquête SUMER, l'absence de lien entre CHSCT et accidents du travail est confirmée même en prenant en compte les expositions professionnelles. Mais à nouveau, cela pourrait s'expliquer par un impact favorable du CHSCT sur ces expositions, hypothèse que nous n'avons pu tester faute de données longitudinales. De même, ne disposant pas d'indications (dans les enquêtes SUMER et Conditions de travail), sur la présence syndicale et le taux de syndicalisation, nous n'avons pas pu étudier en toute généralité l'impact des relations professionnelles sur la qualité des politiques de prévention, et répondre complètement à l'objection présentée par G. Filoche ( $c f$. supra). L'enquête SUMER 2009 permettra d'aller plus loin dans cette direction car elle recueillera des informations plus détaillées sur les relations professionnelles dans l'établissement et sur ses politiques de prévention. Elle permettra également de mieux identifier les liens entre expositions professionnelles, politiques de prévention et santé mentale, du fait de l'introduction d'un questionnaire validé de santé mentale (le questionnaire HAD).

L'absence de lien démontré entre représentation du personnel et santé au travail renvoie aussi à la nature des expositions pour lesquels les CHSCT semblent efficaces: les risques chimiques et biologiques, qui (surtout pour les premiers) ne manifestent leurs effets les plus graves qu'à très long terme. Concernant les expositions qui ont les conséquences les plus immédiates sur la santé, c'est-àdire les contraintes physiques et organisationnelles, le CHSCT semble peu influent. Ce résultat renvoie à la structure classique de la négociation collective en entreprise: les thèmes négociés relèvent des «relations de marché» (salaires, temps de travail...) beaucoup plus que des «relations managériales » (Fox, 1966), qui reposent usuellement sur le présupposé de la subordination du salarié à l'autorité de l'employeur. À cet égard la transformation en 1982, par les lois Auroux, du «comité d'hygiène et de sécurité» (CHS), traditionnellement cantonné aux problèmes d'équipements de sécurité, en «comité d'hygiène, de sécurité et des conditions de travail» destiné à une approche plus globale de la prévention des risques y compris organisationnels, n'a manifestement pas suffi à dépasser ces limites. Comme l'ont déjà montré des travaux monographiques (voir par exemple LinHART et al., 2000), il est moins ardu pour les représentants des salariés de revendiquer des hausses de salaires ou d'exiger la mise en place de protections physiques contre des risques matériels précisément localisés, que de remettre en cause les modes de management et d'organisation du travail. 


\section{Bibliographie}

Burawoy M.(1979), Manufacturing consent, Changes in the Labor Process Under Monopoly Capitalism, The University of Chicago Press.

Burawoy M. (1985), The politics of production. Factory regimes under capitalism and socialism, Verso, London.

Cézard M., Malan A., Zouary P. (1996), «Conflit et régulation sociale dans les établissements », Travail et Emploi, $\mathrm{n}^{\circ} 66$.

Coutrot T. (2002), Critique de l'organisation du travail, La Découverte, Paris.

Coutrot T. (2007), «Plus de trois établissements sur quatre déclarent évaluer les risques professionnels», Premières Synthèses, ${ }^{\circ}$ 2007-09-3, Dares.

Coutrot T. (2008), «La prévention des risques professionnels vue par les salariés», Premières Synthèses, $\mathrm{n}^{\circ}$ 05-1, Dares.

Filoche G. (2001), «Vingt ans de CHSCT», rapport du Conseil économique et social. Site: (http://www.ladocumentationfrancaise.fr/rapports-publics/064000224/ index.shtml).

Fox A. (1966), Industrial Sociology and Industrial Relations, Donovan Commission Research Paper No. 3, HMSO, London.

Frick K., Langaa Jense P., Quinlan M., Wilthagen T. (eds) (2000), Systematic occupational health and safety management - Perspectives on an international development, Pergamon, Oxford.

Garcia A., Lopez-Jacob M.J., Dudzinski I., Gadea R., RoDrIGO F. (2007), "Factors associated with the activities of safety representatives in Spanish workplaces", Journal of Epidemiology and Community Health, 61 : 784-790.

Gollac M. (1998), Donner un sens aux données. L'exemple des enquêtes statistiques sur les conditions de travail, collection "Question de culture», Cahiers du Centre d'étude de l'emploi, Paris, La Documentation française.

Guérin F., Laville A., Daniellou F., Duraffourg J. KerGUELEN A. (2001), Comprendre le travail pour le transformer. La pratique de l'ergonomie, Lyon: ANACT.
Alan H., Forrest A., Sears A., Carlan N. (2006), "Making a difference; knowledge activism and worker representation in joint OHS committees", Relations Industrielles, Summer, Vol. 61, $\mathrm{n}^{\circ} 3$.

Linhart D., Linhart R., Malan A. (1999), « Syndicats et organisation du travail: un jeu de cache-cache?», Travail et Emploi $\mathrm{n}^{\circ} 80$.

Nichols T., Walters D., Tasiran A.C. (2007), "Trade Unions, Institutional Mediation and Industrial Safety: Evidence from the UK", Industrial Relations Society of Australia. Journal of Industrial Relations, 49(2):211225 .

Pignoni M.-T. (2007), «Présence syndicale: des implantations en croissance, une confiance des salariés qui ne débouche pas sur des adhésions », Premières Synthèses, n 2007-14-2, Dares.

Simard M., Carpentier-Roy M.-C., Marchand A., DuelLET F. (1999), Processus organisationnels et psychosociaux favorisant la participation des travailleurs en santé et en sécurité du travail, Études et Recherches, IRSST. http://www.irsst.qc.ca/fr/ projet 257.html.

ThéBAud-Mony A. (1991), La reconnaissance des maladies professionnelles en France. Acteurs et logiques sociales, La Documentation française, Paris.

Walters D., Frick K. (2000), "Worker participation and the management of health and safety: reinforcing or conflicting strategies?", in Frick, Langaa Jense, Quinlan, Wilthagen, 2000.

WALTERS D.(2003), "Workplace arrangements for OHS in the $21^{\text {st }}$ century", National Research Center for OHS regulation, Working paper 10, The Australian National University.

Walters D., Nichol Connor J., Tasiran A.C., Cam S. (2005), The role and effectiveness of safety representatives in influencing workplace health and safety, Health and Safety Executive. http://www.hse.gov.uk/ research/ rrpdf/rr363.pdf.

WOLFF L. (2008), «Le paradoxe du syndicalisme français : un faible nombre d'adhérents, mais des syndicats bien implantés », Premières Synthèses, n 2008-16-1, Dares. 